\title{
TEST ELEMENTS IN EXCELLENT RINGS WITH AN APPLICATION TO THE UNIFORM ARTIN-REES PROPERTY
}

\author{
IAN M. ABERBACH
}

(Communicated by Louis J. Ratliff, Jr.)

\begin{abstract}
Working in positive prime characteristic throughout, we show that excellent rings of dimension 2 or smaller have completely stable test elements and use this to show that excellent domains of dimension 3 have the uniform Artin-Rees property.
\end{abstract}

\section{INTRODUCTION}

By a ring we mean a commutative ring with unit. We use the term local ring to mean a Noetherian ring with unique maximal ideal. If $(R, m)$ is local then we denote the $m$-adic completion of $R$ by $\widehat{R}$.

Let $R$ be a Noetherian ring of positive prime characteristic $p$. Hochster and Huneke have defined the following "tight closure" operation on an ideal $I \subseteq R$ : $x \in R$ is in the tight closure of $I$ if there exists $c$ not in any minimal prime of $R$ such that $c x^{p^{e}} \in\left(i^{p^{e}}: i \in I\right) R$ for all $e$ sufficiently large. This simple definition has led to numerous strong results for rings of equal characteristic. A priori, the element $c$ depends both on $x$ and the ideal $I$. Therefore, it is of interest to find those rings where there are elements that work for all tight closure tests. Such elements are called test elements. Their existence is interesting in its own right and in addition their presence makes possible arguments that have at first glance no connection to tight closure. For instance, Huneke uses test elements to obtain the uniform Artin-Rees property for many rings in [Hu].

If $R$ is a Noetherian domain of characteristic $p$ (or even reduced) then $R$ has an extension containing all $p$ th roots (see below). Given an ideal $I \subseteq R$, we obtain an ideal $I R^{1 / p} \cap R$, which we will call the $p$ th-root closure of $I$. The $p$ th-root closure is contained in the tight closure so if $R$ has a test element $c$ then $c$ multiplies $p$ th-root closure back into the original ideal. Our first result, Theorem 1.2, shows a partial converse to this fact. Under mild conditions on the domain $R$, if $R_{c}$ is regular and $c$ kills $p$ th-root closure then $c^{3}$ is a test element.

Our motivation for proving Theorem 1.2 was to aid in proving that all sufficiently good rings have a test element. Hochster and Huneke have shown that

Received by the editors May 24, 1991 and, in revised form, October 8, 1991.

1991 Mathematics Subject Classification. Primary 13A35; Secondary 13F40.

Key words and phrases. Tight closure, test elements, uniform Artin-Rees.

The author is a NSF Postdoctoral Research Fellow. 
a much stronger statement holds for rings such that $R^{1 / p}$ is a finite $R$-module (see Theorem 1.D) and, by reduction to this case, for all reduced rings of essentially finite type over a sufficiently good local ring (see Theorem 1.A). In Theorem 1.3 we obtain the desired result on the existence of test elements for excellent rings of dimension two or smaller.

In the second section of this paper we apply Theorem 1.3 to obtain a new proof of a result of Huneke's on the uniform Artin-Rees property (in characteristic $p$ ). By developing the theory of test elements in dimension two or less, we can give a new proof that excellent domains of dimension three have the uniform Artin-Rees property. Huneke proves this (with no characteristic conditions) but uses resolution of singularities.

\section{TEST ELEMENTS}

All rings in this section have characteristic $p$, where $p$ is a positive prime integer. We use $e$ to denote a nonnegative integer and $q=p^{e}$ to denote the corresponding power of $p$. For a ring $R$, we use $R^{\circ}$ to denote $R-$ $\bigcup\{$ minimal primes of $R\}$. If $R$ is a domain then by adjoining $p$ th roots for all the elements of $R$ (say in some algebraic closure of the fraction field of $R$ ) we obtain a ring, denoted by $R^{1 / p}$, which contains $R$ as a subring. If $R$ is reduced, with minimal primes $Q_{1}, \ldots, Q_{t}$, then the map $R \rightarrow \prod_{i=1}^{t} R / Q_{i}$ is injective and we obtain $R^{1 / p}$ as the obvious subring of $\prod\left(R / Q_{i}\right)^{1 / p}$.

In [HH2] the following result is proved.

Theorem 1.A. (a) Let $(R, m, k)$ be a local ring of characteristic $p$ such that $R \rightarrow \widehat{R}$ has regular fibers and let $c \in R^{\circ}$ be any element such that $\left(R_{\mathrm{red}}\right)_{c}$ is regular. Then $c$ has a power that is a completely stable $q^{\prime}$-weak test element for $R$.

If $R$ is reduced, then $c$ has a power that is a completely stable test element for $R$.

In particular, if $R$ is an excellent local ring, then $R$ has a completely stable $q^{\prime}$-weak test element; if, moreover, $R$ is reduced, then $R$ has a completely stable test element.

(b) Let $R$ be an algebra of finite type over a local ring $(B, m, k)$ such that $B \rightarrow \widehat{B}$ has smooth fibers (e.g., such that $B$ is excellent). Let $c$ be an element of $R^{\circ}$ such that $\left(R_{\mathrm{red}}\right)_{c}$ is regular. Then $c$ has a power that is a completely stable weak test element for $R$. If $R$ is reduced, then $c$ has a power that is a completely stable test element for $R$.

A ring $R$ is excellent if it is Noetherian, universally catenary, a G-ring (i.e., $R_{p} \rightarrow \widehat{R_{p}}$ is geometrically regular for all primes $P \in \operatorname{Spec}(R)$ ), and the regular locus of any finitely generated $R$-algebra domain is open. For more information see [Ma, Gr].

For convenience we recall the definitions of tight closure and (completely stable) (weak) test elements.

Definition. Let $I \subseteq R$ be an ideal. Then the element $x \in R$, is in the tight closure of $I$, denoted $I^{*}$, if there exists a $c \in R^{\circ}$ such that $c x^{q} \in I^{[q]}$ for all $q \gg 0$, where $I^{[q]}=\left(i^{q}: i \in I\right) R$.

Remark. If $R$ is a domain then for all ideals $I \subseteq R$ the $p$ th-root closure of $I$ 
is contained in the tight closure of $I$. If $x=\sum i_{j} r_{j}^{1 / p}$ (i.e., $x$ is in the $p$ th-root closure of $I$ ) then $1 x^{q}=\sum i_{j}^{q} r_{j}^{q / p} \in I^{[q]}$ so $x \in I^{*}$.

Definition. We say that the ring $R$ is weakly $F$-regular if all ideals of $R$ are tightly closed. We say that $R$ is $F$-regular if $R_{P}$ is weakly F-regular for all primes $P \subseteq R$. We note that [HH1, Theorem 4.6] says that regular rings are F-regular. In this case, every element of $R^{\circ}$ is a test element.

The question of whether there is a single element $c \in R^{\circ}$ that works for every tight closure test (for every ideal) arises naturally and leads to the next definition.

Definition. Let $c \in R^{\circ}$. Then $c$ is said to be a $q^{\prime}$-weak test element if for all ideals $I$ and all elements $x \in I^{*}$ we have $c x^{q} \in I^{[q]}$ for all $q \geq q^{\prime}$. If $q^{\prime}=1$ then we call $c$ a test element. If $c$ is a $q^{\prime}$-weak test element and for all primes $P \in \operatorname{Spec}(R)$ the image of $c$ in $R_{P}$ (resp. $\widehat{R_{P}}$ ) is a $q^{\prime}$-weak test element then $c$ is called a $q^{\prime}$-weak locally stable (resp. $q^{\prime}$-weak completely stable) test element. If, in this case, $q^{\prime}=1$ then $c$ is a locally stable (resp. completely stable) test element.

Remark. [HH1, Proposition 6.1c] shows that if $c$ is a $q^{\prime}$-weak completely stable test element then $c$ is a $q^{\prime}$-weak test element for $R_{P}$ for every prime $P$ in $R$, i.e., $c$ is locally stable. [HH1, Corollary 6.2a] then shows that $c$ is a $q^{\prime}$-weak test element in $U^{-1} R$ for any multiplicative system $U \subseteq R$.

Remark. There is a definition of tight closure for modules using the Frobenius functor (see [HH1, §8]). All the definitions of test element have module counterparts. Theorem 1.A is actually a statement about test elements for modules. With mild hypotheses on the ring (e.g., that all local rings are approximately Gorenstein, see [Ho] - excellent domains are approximately Gorenstein) the two notions of (completely stable) test element coincide (cf. [HH1, (8.6) ff.]). Thus for simplicity we will prove our theorems for ideal tight closure but note once and for all this is sufficient in the cases we are interested in.

We wish to discuss the extension of Theorem 1.A to the global case, but first we note some general reductions concerning test elements. The next two results are in $\S 6$ of [HH1] (cf. (6.1) and (6.25) there).

Proposition 1.B. Let $R$ be Noetherian of characteristic $p$ and $c \in R$. Then $c$ is a $q^{\prime}$-weak test element for $R$ iff $c / 1$ is a $q^{\prime}$-weak test element for $R_{m}$ for every maximal ideal $m$ of $R$.

Proposition 1.C. Let $R$ be a Noetherian ring of characteristic $p$ with minimal primes $P_{1}, \ldots, P_{t}$. Let $N=P_{1} \cap \cdots \cap P_{t}$ be the nilradical and suppose that $N^{\left[q^{\prime \prime}\right]}=0$. If each of the rings $R / P_{i}$ has a $q^{\prime}$-weak test element then $R$ has a $q^{\prime} q^{\prime \prime}$-weak test element. Hence, if $R$ is reduced and each $R / P_{i}$ has a test element then $R$ has a test element.

Remark. An immediate consequence of Proposition 1.B and the definition of $q^{\prime}$-weak completely stable test element is that Proposition 1.B holds for $q^{\prime}$-weak completely stable test elements (the only if part becomes trivial since it is part of the definition of completely stable). The same is true for Proposition 1.C.

By virtue of Proposition 1.C we will be concerned only with obtaining test elements for domains. 
We review briefly the proof of Theorem 1.A (in the local case). The hypothesis that $R \rightarrow \widehat{R}$ has regular fibers allows passage to the complete case because then $\widehat{R}_{c}$ is also regular and Lemma 1 .E reduces the claim to proving $c$ works for $\widehat{R}$. The key step at this point is to extend a coefficient field of $R$ to get a map $(R, m) \rightarrow\left(R^{\prime}, m^{\prime}\right)$ in such a way that $R^{\prime}$ is a faithfully flat, purely inseparable extension of $R$ with Gorenstein fibers, the ramification of primes is controlled, and the Frobenius endomorphism of $R^{\prime}$ is a module-finite map. This allows the application of the following theorem to $R^{\prime}$. (See [HH3] for a definition of strongly F-regular - the point is that regular rings are strongly $F$ regular [HH3, Theorem 3.1c], so that if $c$ is in the defining ideal of the singular locus for $R$ then $R_{c}$ is strongly F-regular.)

Theorem 1.D [HH3]. Let $R$ be a Noetherian domain of characteristic $p$ such that $R^{1 / p}$ is module-finite over $R$. Then every element $c^{\prime} \in R^{\circ}$ such that $R_{c^{\prime}}$ $i$ strongly $F$-regular has a power that is a test element.

More precisely, $c^{\prime}$ has a power $c$ such that there is an $R$-linear map $h: R^{1 / p} \rightarrow$ $R$ that sends 1 to $c$, and for $c \in R^{\circ}$ with this property such that $R_{c}$ is strongly $F$-regular, $c^{3}$ is a test element.

Note that given such a $c$ and map $h$ (whether or not $R^{1 / p}$ is finite over $R$ ) we get that $c\left(I R^{1 / p} \cap R\right) \subseteq I$ for every ideal $I \subseteq R$. Write $x=\sum i_{j} r_{j}^{1 / p}$ and then

$$
x c=x h(1)=h(x)=h\left(\sum i_{j} r_{j}^{1 / p}\right)=\sum i_{j} h\left(r_{j}^{1 / p}\right) \in I .
$$

This fact suggests trying to prove that if an element in the defining ideal of the singular locus kills $p$ th-root closure (which recall is contained in tight closure) then its cube is a test element. Following two lemmas, we show in Theorem 1.2 that this is true in an excellent ring and the test element is completely stable.

Lemma 1.E [HH2]. Suppose that $R \subseteq S$ is faithfully flat and that $c \in R^{\circ}$ is a completely stable $q^{\prime}$-weak test element for $S$. Then $c$ is a completely stable $q^{\prime}$-weak test element for $R$.

Lemma 1.1. Let $R$ be an analytically unramified local ring (i.e., $\widehat{R}$ is reduced) and assume $c \in R$ kills pth-root closure for all $m$-primary ideals in $R$. Then $c$ has the same property for $\widehat{R}$.

Proof. Every $m \widehat{R}$-primary ideal is extended from $R$. Let $J=I \widehat{R}$ be $m \widehat{R}$ primary where $I \subseteq R$ is $m$-primary. Then $K=J \widehat{R}^{1 / p} \cap \widehat{R}$ is also $m \widehat{R}$-primary so is extended from $R$, say $K=W \widehat{R}$. If $w \in W$ then $w^{p} \in J^{[p]} \widehat{R} \cap R=I^{[p]}$. Thus $w \in I R^{1 / p} \cap R$. Hence, $c K=c W \widehat{R} \subseteq c\left(I R^{1 / p} \cap R\right) \widehat{R} \subseteq I \widehat{R}=J$.

Theorem 1.2. Let $R$ be a Noetherian domain of characteristic $p$. Assume that for all maximal ideals $m$ such that $R_{m}$ is not regular the map $R_{m} \rightarrow \widehat{R_{m}}$ is regular, e.g., $R$ excellent. Let $c \in R^{\circ}$ be such that $R_{c}$ is regular and suppose that for all ideals $I \subseteq R$ primary to a maximal ideal we have $c\left(I R^{1 / p} \cap R\right) \subseteq I$. Then $c^{3}$ is a completely stable test element.

Proof. By Proposition 1.B and the ensuing remark we need to show that $c^{3} / 1$ is a completely stable test element in $R_{m}$ for every maximal ideal of $R$. Note that the given condition for $c$ localizes at maximal ideals because taking $p$ th roots 
commutes with localization and all $m R_{m}$-primary ideals are extended from $R$. If $R_{m}$ is regular then every nonzero element is a completely stable test element.

Thus we have reduced to the case of $(R, m)$ a local domain such that the map to the completion is regular. By [Ma, (33.B), Lemma 2], $\widehat{R}$ is reduced. By Lemma 1.1 the element $c$ kills $p$ th-root closure for all $m \widehat{R}$-primary ideals. By Lemma 1.E we need to show that $c^{3}$ is a completely stable test element in $\widehat{R}$. Hence we may assume that $(R, m)$ is a complete, reduced local ring, $R_{c}$ is regular, and $c$ kills $p$ th-root closure for all $m$-primary ideals. It is easy to see that this last condition ensures that $c$ kills $p$ th-root closure for all ideals $I \subseteq R$. Let $x \in I R^{1 / p} \cap R$. Then for all $l>0, x \in\left(I+m^{l}\right) R^{1 / p} \cap R$ so $c x \in \bigcap_{l}\left(I+m^{l}\right)=I$.

The property that $c$ kills $p$ th-root closure commutes with localization, and since $R$ is complete, it is excellent. Thus $\left(\widehat{R_{P}}\right)_{c}$ is regular [Ma, (33.E), Lemma 4 and (33.B), Lemma 2], and by our above arguments the image of $c$ in $\widehat{R_{P}}$ kills $p$ th-root closure, for every prime $P$ in $R$. Renaming $\widehat{R_{P}}$ as $R$ again we need to show that $c^{3}$ is a test element.

We claim that $c^{2}\left(I R^{1 / q} \cap R\right) \subseteq I$ for all ideals $I$ and for all $q \geq 0$. This can be seen by induction on $e$, where $q=p^{e}$. The case $e=1$ is our hypothesis. Now assume that the claim holds for $q^{\prime}$ (for all ideals) and let $q=p q^{\prime}$. Let $x \in I R^{1 / q} \cap R$. Then $x^{p} \in I^{[p]} R^{1 / q^{\prime}} \cap R$ so by our induction hypothesis $c^{2} x^{p} \in$ $I^{[p]}$. Hence $c^{p} x^{p} \in I^{[p]}$ and taking $p$ th roots yields that $c x \in I R^{1 / p} \cap R$. Thus $c(c x)=c^{2} x \in I$.

To complete the proof of the first statement let $x \in I^{*}$. By Theorem 1.A some power (which we may assume is a power of $p$ ) of $c$ is a test element, say $c^{q_{0}}$. Then $c^{q_{0}} x^{q_{0}} \in I^{\left[q_{0}\right]}$ from which it follows that $c x \in I R^{1 / q_{0}} \cap R$. Now, by the above claim $c^{2}(c x)=c^{3} x \in I$.

Thus, one approach to finding global test elements is to find such elements described above. More specifically, we would like to show that every element in the defining ideal of the singular locus of an excellent domain has a power that kills $p$ th-root closures for all ideals. Unfortunately this approach seems no more tractable than looking for test elements outright.

In excellent rings of small dimension we do have test elements as the following theorem shows. (The essence of the argument for dimension two is Hochster's.)

Theorem 1.3. Let $R$ be an excellent domain of dimension less than or equal to 2. Let $I$ be the defining ideal of the singular locus. Then every nonzero element of I has a power that is a completely stable test element.

Proof. If $\operatorname{dim} R=0$ then $R$ is a field (thus regular) and every nonzero element is a test element. If $\operatorname{dim} R=1$ then $I \neq 0$ is contained in only finitely many maximal ideals, say $m_{1}, \ldots, m_{t}$. Let $c \in I$. For $m \notin\left\{m_{1}, \ldots, m_{t}\right\}, R_{m}$ is regular, hence F-regular, so $c / 1$ is a completely stable test element. For each $m_{i}$, some power of $c / 1$ is a completely stable test element for $R_{m}$ by Theorem 1.A, so taking $c$ to the maximal such power and applying Proposition 1.B we see that some power of $c$ is a completely stable test element for $R$.

Suppose that $R$ has dimension 2. Let $S$ be the integral closure of $R . S$ is a module-finite $R$-module because $R$ is excellent. Let $J \subseteq S$ be the defining ideal of the singular locus. Then ht $J \geq 2$ since the normality of $S$ ensures 
that $S_{P}$ is a discrete valuation domain for all height one primes of $S$. By a similar argument to the one given above ( $J$ is contained in only finitely many maximal ideals of $S$ ), every element of $J$ has a power that is a completely stable test element for $S$.

Now, let $c \in I$. Then $R_{c}$ is regular, hence integrally closed, thus the map $R_{c} \rightarrow S_{c}$ is an isomorphism. Thus $S_{c}$ is regular and hence $c \in J$. Because $S$ is a finite $R$-module there is a power of $c$, say $c^{l}$ such that $c^{l} S \subseteq R$ and (possibly enlarging the power) we may assume that $c^{l}$ is a completely stable test element for $S$. To simplify notation we replace $c^{l}$ by $c$. Now let $W \subseteq R$ be an ideal and suppose that $x \in W^{*}$. Then $x \in(W S)^{*}$ so $c x \in W S$. Thus $c(c x)=c^{2} x \in W$. Hence $c^{2}$ is a test element for $R$. In particular $c^{2}$ kills $p$ th-root closure for $R$ so by Theorem $1.2\left(c^{2}\right)^{3}=c^{6}$ is a completely stable test element for $R$.

From the proof of Theorem 1.3 it is clear that when trying to prove this statement in higher dimensions we may pass to the integral closure. Thus the first open case is a three-dimensional excellent normal domain whose singular locus has height two.

Remark. The assumption of excellence in Theorem 1.3 can be weakened; however, unlike Theorem 1.A, more than just the assumption of regular fibers is necessary (for our proof) as we use the finiteness of integral closure.

\section{The UNIFORM ARTIN-REES PROPERTY FOR 3-DIMENSIONAL DOMAINS}

Definitions. We say that the Noetherian ring $R$ has the uniform Artin-Rees property if for every pair of finitely generated $R$-modules $N \subseteq M$, there exists an integer $k=k(M, N)$ such that for all $I \subseteq R$ and for all $n \geq k, I^{n} M \cap N \subseteq$ $I^{n-k} N$. We say then that $N \subseteq M$ has uniform number $k$.

Huneke's paper [Hu] shows that many rings satisfy the uniform Artin-Rees property, for instance algebras essentially of finite type over local rings [Hu, Theorem 4.12]. A slightly different uniform Artin-Rees condition concerning bounds for all maximal ideals is demonstrated for excellent rings in [DO].

Both Theorem 1.A and [Hu, Theorem 4.12] are results for local rings that ought to hold for excellent global rings. Given $R$ excellent (and an element $c \in R$ such that $R_{c}$ is regular if we are trying to obtain test elements or $N \subseteq M$ finitely generated $R$-modules if we are investigating the uniform Artin-Rees property) we may apply each theorem for $R_{m}$ for each maximal ideal $m$ to obtain an integer $k(m)$ (where $c^{k(m)}$ is a completely stable test element for $R_{m}$ or for all $R_{m}$-ideals $\left.I_{m}^{t} M_{m} \cap N_{m} \subseteq I_{m}^{t-k(m)} N_{m}\right)$. If, given a maximal ideal $m$ we could find a small enough open set $U \subseteq \operatorname{MaxSpec}(R)$ such that $k\left(m^{\prime}\right) \leq k(m)$ for all $m^{\prime} \in U$, then we could use the quasi-compactness of the Zariski topology on $\operatorname{Max} \operatorname{Spec}(R)$ to obtain a global bound.

Theorem 1.3 can be used to give a new proof of [Hu, Theorem 5.11] in the positive characteristic case. We state the theorem and sketch a proof below.

Theorem 2.1 [Hu]. Let $R$ be an excellent three-dimensional domain. Then $R$ has the uniform Artin-Rees property.

Theorem 2.1 follows from [Hu, Remark 4.11], which reduces to the case that $R$ has infinite residue fields, and 
Theorem 2.2 [cf. $\mathrm{Hu}$, Theorem 3.4]. Let $R$ be a universally catenary Noetherian ring of finite Krull dimension having infinite residue fields. Assume that for all prime ideals $P \subseteq R, R / P$ has a completely stable test element. Then $R$ has the uniform Artin-Rees property. Furthermore, if $R$ is a domain then we need only assume that $R / P$ has the above properties for all nonzero primes.

In order to make this note somewhat self-contained we introduce some notation and sketch several proofs from [Hu].

Definition. Let $R$ be a Noetherian ring. For $k \geq 0$ define $T_{k}(R)=\bigcap_{n, I} I^{n-k}$ : $\overline{\left(I^{n}\right)}$, where the intersection is over all $n \geq 0$ and all $I \subseteq R$ and $\bar{J}$ is the integral closure of $J$. Now we define $T(R)=\bigcup_{k} T_{k}(R)$.

The connection between test elements and the ideal $T(R)$ is given by the next theorem.

Proposition 2.B [Hu, Proposition 4.7i and proof]. Suppose the reduced Noetherian ring $R$ has characteristic $p$ and is finite dimensional. Assume that $R$ has a test element $c$. Then $c \in T(R)$.

We need to discuss the concept of phantom homology in [HH1, HH4]. First we will need several definitions.

Definition. Let $R$ be a Noetherian ring. The minheight of $I \subseteq R$, $\operatorname{minht}_{R} I$ is $\min \{\mathrm{ht} I(R / P): P$ is a minimal prime of $R\}$.

Remark. [HH4, Proposition 2.2f] says that if $R$ is universally catenary and $S$ is faithfully flat over $R$ then $\operatorname{minht}_{R} I=\operatorname{minht}_{S} I S$.

Definitions. Let $\left(G_{\bullet}, f_{\bullet}\right)$ be a finite complex of finitely generated free modules. By $I\left(f_{i}\right)$ we mean the ideal generated by the determinantal rank size minors of a matrix of $f_{i}$ (this is independent of the matrix). We say that $G_{0}$ satisfies the standard conditions on rank and minheight if rank $f_{i}+\operatorname{rank} f_{i-1}=\operatorname{rank} G_{i}$ and minht $I\left(f_{i}\right) \geq i$ for $i \geq 1$. (For more information, see [HH1, §§9.6, 9.7; HH4, $\S 2]$.) Note that the Buchsbaum-Eisenbud criterion for exactness [BE] says that $G$. is acyclic if and only if $G$. satisfies the standard conditions on rank and depth.

[HH4, Theorem 3.22], the phantom acyclicity criterion, says (a great deal more than) that if $R$ is a finite-dimensional image of a Gorenstein ring and if $G . \otimes R_{\text {red }}$ satisfies the standard conditions on rank and minheight then any test element $c$ for $R$ kills $H_{i}\left(G_{\bullet}\right)$ for $i \geq 1$.

The next lemma may be considered an addition to the list given in $[\mathrm{Hu}$, Proposition 4.5].

Lemma 2.3. Let $R$ be a universally catenary Noetherian domain with a completely stable test element $c$. Let $G_{0}$. be a finite complex of finitely generated free modules satisfying the standard conditions on rank and height. Then $c H_{i}\left(G_{\bullet}\right)=0$ for all $i \geq 1$.

Proof. The question is local on maximal ideals so we may assume that $R$ is local. Since $R$ is universally catenary $\operatorname{minht} I \widehat{R}=\operatorname{minht} I$ (which equals ht $I$ because $R$ is a domain) for all ideals $I \subseteq R$. Thus $G_{\bullet} \otimes(\widehat{R})_{\text {red }}$ satisfies the standard conditions on rank and height so $c H_{i}\left(G_{\bullet} \otimes \widehat{R}\right)=0$. By the faithful flatness of $\widehat{R}, c H_{i}\left(G_{\bullet}\right)=0$ for $i \geq 1$. 
Lemma 2.4 (cf. [Hu, Lemma 3.3]). Let $R=S / P$ where $S$ is universally catenary. Assume that the image of $c \in S$ is a completely stable test element for $R$. Also, assume that the pair $P+c S \subseteq S$ satisfies the uniform Artin-Rees property with uniform number $k$. Let $J$ be any ideal of $S$ generated by elements $a_{1}, \ldots, a_{d}$ such that ht $J R \geq d$. Then $J^{n} \cap P \subseteq J^{n-k} P$ for all $n \geq k$.

Proof. The $n$th power of $J$ is given by the size $n$ minors of an $n$ by $n+d-1$ matrix (cf. [BV, Remark 2.13])

$$
B=\left(\begin{array}{ccccccc}
a_{1} & a_{2} & \cdots & a_{d} & 0 & \cdots & 0 \\
0 & a_{1} & \cdots & a_{d-1} & a_{d} & \cdots & 0 \\
\vdots & & & & & & \vdots \\
0 & 0 & \cdots & \cdots & \cdots & a_{d-1} & a_{d}
\end{array}\right) .
$$

In [EN] a free complex $\left(G_{\bullet}, f_{\bullet}\right)$ of length $(n+d-1)-n+1=d$ is constructed such that $H_{0}\left(G_{\bullet}\right)=S / J^{n}$. The nonzero entries of the matrices for $f$ involve only the generators of $J$. In fact, one may show that $\sqrt{I\left(f_{i}\right)}=J$. The complex satisfies the standard condition on rank because it does so generically [EN, Theorem 2; BE, Lemma 5]. Hence the Eagon-Northcott complex for $J^{n} R$ satisfies the standard rank and height conditions. Thus by Lemma 2.3, $c$ kills $H_{1}\left(G_{\bullet}\right)$. By the right exactness of tensor, $H_{1}\left(G_{\bullet}\right)$ maps onto $\operatorname{Tor}_{1}^{S}\left(S / J^{n}, S / P\right)$, and hence $c$ kills this module also. Reinterpteting this says that $c\left(J^{n} \cap P\right) \subseteq J^{n} P$ for all $n \geq 1$. Thus for all $n \geq k$

$$
\begin{aligned}
J^{n} \cap P \subseteq J^{n} \cap(P+c S) \cap P & \subseteq J^{n-k}(P+c S) \cap P \\
& \subseteq\left(J^{n-k} P+J^{n-k} c\right) \cap P \\
& =J^{n-k} P+c\left(J^{n-k} \cap P\right)=J^{n-k} P .
\end{aligned}
$$

We now outline a proof of Theorem 2.2 corresponding to Huneke's proof of [Hu, Theorem 3.4]. It is easy to reduce to showing that the uniform Artin-Rees property holds for all pairs $P \subseteq R$ where $P$ is prime [Hu, Proposition 2.2]. By induction on the dimension of the quotient we may assume that uniform ArtinRees holds for all $N \subseteq M$ such that $\operatorname{dim} M / N<\operatorname{dim} R / P$. In particular, let $c \in R$ be an element whose image in $R / P$ is a test element. Then the uniform Artin-Rees property holds for $P+c R \subseteq R$, say with uniform number $k$. We use ' to denote images in $R^{\prime}=R / P$. By Proposition 2.B we know that $c^{\prime} \in T_{t}(R / P)$ for some $t$. We claim that the pair $P \subseteq R$ has uniform number $2 k+t+1$.

[Hu, Proposition 2.3] shows that we only need to show the uniform ArtinRees property for ideals primary to a maximal ideal. Let $I$ be $m$-primary. Let $l=\operatorname{dim} R_{m}^{\prime}$. Then we can find $K \subseteq R$ such that $K R_{m}^{\prime}$ is a minimal reduction of $I R_{m}^{\prime}$ and elements $a_{1}, \ldots, a_{l} \in K$ such that if $J=\left(a_{1}, \ldots, a_{l}\right)$ then $J R_{m}^{\prime}=K R_{m}^{\prime}$ and ht $J R^{\prime} \geq l$. Now Proposition 2.4 shows that $J^{n} \cap P \subseteq J^{n-k} P$ for all $n \geq k$.

Note that $K^{\prime} \subseteq I^{\prime}$ and they have the same integral closure (they were chosen this way in $R_{m}^{\prime}$ and the same is true in $R^{\prime}$ since they are $m^{\prime}$-primary). Since $c^{\prime}$ is a nonzero element of $T_{t}\left(R^{\prime}\right), c^{\prime} \overline{\left(I^{\prime n}\right)} \subseteq\left(K^{\prime}\right)^{n-t} \cap\left(c^{\prime}\right) \subseteq\left(K^{\prime}\right)^{n-t-k} c^{\prime}$ (the last containment follows from uniform Artin-Rees for $P+c R \subseteq R$ ). Thus $\overline{\left(I^{\prime n}\right)} \subseteq\left(K^{\prime}\right)^{n-t-k}$, from which we may conclude that $\left(I^{n}\right)_{m} \subseteq\left(J^{n-t-k}\right)_{m}+P_{m}$ for all $n \geq t+k$. 
We may now apply [Hu, Lemma 3.1] to $R_{m}$ and then using the fact that $I$ is $m$-primary, obtain the uniform Artin-Rees property for the pair $P \subseteq R$.

Putting together Theorems 1.3 and 2.2 yields the proof of Theorem 2.1.

\section{ACKNOWLEDGMENT}

The author wishes to thank Mel Hochster for helpful conversations and Craig Huneke both for suggesting the second section of this paper and other helpful conversations.

\section{REFERENCES}

[BE] D. Buchsbaum and D. Eisenbud, What makes a complex exact?, J. Algebra 25 (1973), 259-268.

[DO] A. J. Duncan and L. O'Carroll, A full uniform Artin-Rees theorem, J. Reine Angew. Math. 394 (1989), 203-207.

[EN] J. A. Eagon and D. G. Northcott, Ideals defined by matrices and a certain complex associated with them, Proc. Roy. Soc. London Ser. A 269 (1962), 188-204.

[Gr] A. Grothendieck, Éléments de géométrie algébrique IV $_{2}$, Inst. Hautes Études Sci. Publ. Math. 20 (1964).

[Ho] M. Hochster, Cyclic purity versus purity in excellent Noetherian rings, Trans. Amer. Math. Soc. 231 (1977), 463-488.

[HH1] M. Hochster and C. Huneke, Tight closure, invariant theory, and the Briançon-Skoda Theorem, J. Amer. Math. Soc. 3 (1990), 31-116.

[HH2] _ F-regularity, test elements and smooth base change rings, preprint.

[HH3] _ Tight closure and strong F-regularity, Numéro consacré ou colloque en l'honneur de P. Samuel, Mém. Soc. Math. de France (N.S), no. 38 (1989), 119-133.

[HH4] _ Phantom homology, preprint.

[Hu] C. Huneke, Uniform bounds in Noetherian rings, Invent. Math. (to appear).

[Ma] H. Matsumura, Commutative algebra, Benjamin/Cummings, Cambridge, New York, New Rochelle, Melbourne, Sydney, 1980.

Department of Mathematics, Purdue University, West Lafayette, Indiana 47907

E-mail address: ian@math.purdue.edu 